\title{
A uniform estimate for quartile operators
}

\section{Christoph Thiele}

\begin{abstract}
There is a one parameter family of bilinear Hilbert transforms. Recently, some progress has been made to prove $L^{p}$ estimates for these operators uniformly in the parameter. In the current article we present some of these techniques in a simplified model: we prove the estimate $L^{2} \times L^{2} \rightarrow L^{1, \infty}$ uniformly for a discrete family of model operators for the bilinear Hilbert transform.
\end{abstract}

\section{Introduction}

The quartile operator, introduced in [10],[11], is a discrete model for the bilinear Hilbert transform. The latter is a bilinear operator on the product of Schwartz classes $S(\mathbb{R}) \times S(\mathbb{R})$ defined by

$$
H_{\alpha}\left(f_{1}, f_{2}\right)(x):=p \cdot v \cdot \int f_{1}(x+t) f_{2}(x+\alpha t) \frac{d t}{t} .
$$

Here $\alpha \neq 0,1$ is a real parameter. In the series of articles [5], [6], [7], and [8], it has been proved that the bilinear Hilbert transform satisfies the $L^{p}$ estimate

$$
\left\|H_{\alpha}\left(f_{1}, f_{2}\right)\right\|_{\frac{p_{1} p_{2}}{p_{1}+p_{2}}} \leq C\left(\alpha, p_{1}, p_{2}\right)\left\|f_{1}\right\|_{p_{1}}\left\|f_{2}\right\|_{p_{2}}
$$

provided $1<p_{1}, p_{2} \leq \infty$ and $2 / 3<\frac{p_{1} p_{2}}{p_{1}+p_{2}}<\infty$. However, this series of articles gives constants $C\left(\alpha, p_{1}, p_{2}\right)$ which tend to $\infty$ as $\alpha$ approaches 0,1 or $\infty$.

Since $H_{0}$ (or $H_{1}$, or $H_{\infty}$ with appropriate interpretation) is a composition of the linear Hilbert transform and a pointwise product, it is easy to see that

2000 Mathematics Subject Classification: 42A50.

Keywords: Bilinear Hilbert Transform, Multilinear Singular Integrals, Uniform Estimates. 
$H_{0}$ satisfies the above $L^{p}$ estimate for certain values of $p_{1}, p_{2}$. It is natural to conjecture that for these values of $p_{1}, p_{2}$ the constant $C\left(\alpha, p_{1}, p_{2}\right)$ can be chosen uniformly as $\alpha$ approaches 0 . A first step towards this conjecture is done in [13], where the weak type estimate $L^{2} \times L^{2} \rightarrow L^{1, \infty}$ is proved uniformly in $\alpha$ as long as $\alpha$ is contained in $\left[-\frac{1}{2}, \frac{1}{2}\right]$. I am indebted to Xiaochun $\mathrm{Li}$ for pointing out to me that an argument in [13] deducing strong type estimates from this weak type estimate is not correct. In the process of publication of the current article, two preprints [3] by Grafakos and Li and [9] by Li have become available which prove uniform bounds for the bilinear Hilbert transform in a wide range of exponents.

The purpose of this article is to provide a Walsh model for the proof of uniform estimates. Thus we define a family $H_{L}$ of modified quartile operators and prove uniform weak type estimates $L^{2} \times L^{2} \rightarrow L^{1, \infty}$ for the operators $H_{L}$. This proof contains most of the important ideas used to prove the uniform estimate for the bilinear Hilbert transform, but many technical issues disappear because of the sharp time frequency localization of the Walsh wave packets involved. The use of Walsh models to present simplified counterparts of theorems in Fourier analysis has precedents: [1] for [2], [11] for [5], and [12] for [4].

In this paper a dyadic interval is one of the form $\left[2^{k} n, 2^{k}(n+1)\right)$ with $k, n \in \mathbb{Z}$ and $n \geq 0$. Let $C^{\Delta}\left(\mathbb{R}^{+}\right)$denote the set of all functions $f: \mathbb{R} \rightarrow$ $\mathbb{R}$ that are finite linear combinations of characteristic functions of dyadic intervals (and thus vanish outside the positive half axis). For $l \geq 0$ we define the $l$-th Walsh function $W_{l} \in C^{\Delta}\left(\mathbb{R}^{+}\right)$by the following recursive formulas:

$$
\begin{aligned}
W_{0} & =1_{[0,1)}, \\
W_{2 l} & =W_{l}(2 x)+W_{l}(2 x-1), \\
W_{2 l+1} & =W_{l}(2 x)-W_{l}(2 x-1) .
\end{aligned}
$$

A tile is a rectangle $I_{p} \times \Omega_{p}$ of area one, such that $I_{p}$ and $\Omega_{p}$ are dyadic intervals. If $p=\left[2^{k} n, 2^{k}(n+1)\right) \times\left[2^{-k} l, 2^{-k}(l+1)\right)$ is a tile, we define the corresponding Walsh wave packet $w_{p}$ by

$$
w_{p}(x)=2^{-\frac{k}{2}} W_{l}\left(2^{-k} x-n\right)
$$

Moreover we define the Haar function $h_{p}$, which really depends only on the parameters $k$ and $n$ of $p$, as

$$
h_{p}(x)=2^{-\frac{k}{2}} W_{1}\left(2^{-k} x-n\right) .
$$


For each integer $L \geq 1$ and $f_{1}, f_{2}, f_{3} \in C^{\Delta}\left(\mathbb{R}^{+}\right)$define $\Lambda_{L}\left(f_{1}, f_{2}, f_{3}\right)$ to be

$$
\int \sum_{\Omega}\left(\sum_{p: \Omega_{p}=\Omega_{u}}\left\langle f_{1}, w_{p}\right\rangle h_{p}(x)\right) \prod_{j=2}^{3}\left(\sum_{p: \Omega_{p}=2^{L} \Omega_{l}}\left\langle f_{j}, w_{p}\right\rangle w_{p}(x)\right) d x .
$$

Here we have used the following notation: if $\Omega=[\xi, \eta)$ is a dyadic interval, then $\Omega_{l}$ is the lower half $[\xi,(\xi+\eta) / 2), \Omega_{u}$ is the upper half $\Omega \backslash \Omega_{l}$, and $2^{L} \Omega:=\left[2^{L} \xi, 2^{L} \eta\right)$. The summation is over all dyadic intervals $\Omega$.

Consider the dual bilinear operator to $\Lambda_{L}$ mapping $C^{\Delta}\left(\mathbb{R}^{+}\right) \times C^{\Delta}\left(\mathbb{R}^{+}\right)$ to locally integrable functions defined by

$$
\int H_{L}\left(f_{1}, f_{2}\right)(x) f_{3}(x) d x=\Lambda_{L}\left(f_{1}, f_{2}, f_{3}\right) .
$$

Then we have the following theorem:

Theorem 1.1 $H_{L}$ extends to a bounded operator from $L^{2} \times L^{2}$ into $L^{1, \infty}$ uniformly in $L \geq 1$, i.e., there is a contstant $C$ independent of $L$ such that

$$
\left|\left\{x:\left|H_{L}\left(f_{1}, f_{2}\right)(x)\right|>\lambda\right\}\right| \leq C \lambda^{-1}\left\|f_{1}\right\|_{2}\left\|f_{2}\right\|_{2}
$$

for all $f_{1}, f_{2} \in C^{\Delta}\left(\mathbb{R}^{+}\right)$.

We do not intend a detailed explanation why the forms $\Lambda_{L}$ are a natural discrete analogue of the family of forms $\Lambda_{\alpha}$ associated to the bilinear Hilbert transforms $H_{\alpha}$. We just mention that morally (modulo some technical issues) the forms $\Lambda_{\alpha}$ can be written as

$$
\int \sum_{\Omega} \prod_{j=1}^{3}\left(f_{j} * \phi_{A_{j, \alpha} \Omega}\right) d x
$$

where $A_{j, \alpha}$ are certain affine transformations and the Fourier transform of the function $\phi_{A_{j, \alpha} \Omega}$ is supported in the interval $A_{j, \alpha} \Omega$. These affine transformations are modelled by the transformations in the definition of $\Lambda_{L}$ : the appearance of an $h_{p}$ in the definition of $\Lambda_{L}$ becomes necessary because the product structure of Walsh functions is different from the product structure of exponentials.

Part of the work on this article has been done during a delightful stay at the Erwin Schrödinger Institute in Vienna during the summer of 1999. The author was also supported by NSF grant DMS 9970469. The author would like to thank C. Muscalu and T. Tao for many helpful discussions improving the final version of this paper. 


\section{The truncated trilinear form}

If $I_{p} \times \Omega_{p}$ is a tile, we call $I_{p}$ the spatial interval and $\Omega_{p}$ the frequency interval of $p$. Generally we will keep a distinction between spatial intervals $I$ and frequency intervals $\Omega$.

For notational convenience we fix $L$ and write $\Lambda$ and $H$ for $\Lambda_{L}$ and $H_{L}$. Furthermore, for any dyadic frequency interval $\Omega$ we write

$$
\begin{gathered}
\Omega f:=\sum_{p: \Omega_{p}=\Omega}\left\langle f, w_{p}\right\rangle h_{p}, \\
\bar{\Omega} f:=\sum_{p: \Omega_{p}=2^{L} \Omega}\left\langle f, w_{p}\right\rangle w_{p} .
\end{gathered}
$$

Since we are considering real valued functions only, there is no danger of confusing the notation $\bar{\Omega}$ with complex conjugation. The reader should be alerted that the spatial scale associated with $\bar{\Omega}$ is $2^{-L}$ times the spatial scale that one might expect. Thus we can write the trilinear form $\Lambda$ as

$$
\Lambda\left(f_{1}, f_{2}, f_{3}\right)=\int \sum_{\Omega}\left(\Omega_{u} f_{1}\right)(x) \prod_{j=2}^{3}\left(\overline{\Omega_{l}} f_{j}\right)(x) d x .
$$

For a spatial set $E \subset \mathbb{R}^{+}$and an integer $k$ define $E_{k}$ to be the union of all dyadic intervals of length $2^{k}$ which have nonempty intersection with $E$. Observe that we have the nesting property

$$
E_{k} \subset E_{k^{\prime}} \quad \text { if } \quad k<k^{\prime} .
$$

We write

$$
E_{k} f(x)=\left\{\begin{array}{cl}
f(x) & \text { if } x \in E_{k} \\
0 & \text { otherwise }
\end{array}\right.
$$

and we shall use the same convention for any spatial subset of $\mathbb{R}^{+}$. We generally reserve the letters $E, F$, and $I$ for spatial sets and the letter $\Omega$ for frequency sets, so there should be no confusion between the definitions (2.1) and (2.4).

For any spatial set $E$ define the truncated form $\Lambda_{E}$ by

$$
\Lambda_{E}\left(f_{1}, f_{2}, f_{3}\right)=\int \sum_{k} \sum_{|\Omega|=2^{1-k}}\left(E_{k-L} \Omega_{u} f_{1}\right)(x) \prod_{j=2}^{3}\left(\overline{\Omega_{l}} f_{j}\right)(x) d x .
$$

Let $M_{2}$ denote the variant of the Hardy Littlewood maximal operator defined by $L^{2}$ - means. 
Lemma 2.1 Let $f_{j} \in C^{\Delta}\left(\mathbb{R}^{+}\right)$with $\left\|f_{j}\right\|_{2}=1$ for $j=1,2,3$. Define

$$
E:=\bigcap_{j=1}^{3}\left\{M_{2} f_{j}(x) \leq 1\right\}
$$

Then

$$
\left|\Lambda_{E}\left(f_{1}, f_{2}, f_{3}\right)\right| \leq C
$$

for some constant $C$ independent of $f_{j}$ and $L$.

We postpone the proof of Lemma 2.1 and prove that Lemma 2.1 implies Theorem 1.1.

Let $f_{1}, f_{2} \in C^{\Delta}\left(\mathbb{R}^{+}\right)$with $\left\|f_{1}\right\|_{2}=\left\|f_{2}\right\|_{2}=1$. By linearity and dilation invariance it suffices to prove

$$
\left|\left\{H\left(f_{1}, f_{2}\right)(x)>C\right\}\right| \leq C .
$$

Define

$$
E:=\bigcap_{j=1}^{2}\left\{M_{2} f_{j}(x) \leq 1\right\} .
$$

Clearly $\left|E^{c}\right| \leq C$. Let $H_{E}$ be the dual operator to $\Lambda_{E}$ in analogy to (1.4). Since $E \subset E_{k}$ for all $k$, we have that $H\left(f_{1}, f_{2}\right)$ coincides with $H_{E}\left(f_{1}, f_{2}\right)$ on $E$. Here we use that $\overline{\Omega_{l}}$ acts locally on all dyadic intervals of length $2^{k-L}$ where $|\Omega|=2^{1-k}$. We point out that we could not do this argument if we had dualized in the first function $f_{1}$ rather than in the third function $f_{3}$.

Thus it suffices to prove that

$$
\left|\Lambda_{E}\left(f_{1}, f_{2}, f_{3}\right)\right| \leq C
$$

whenever $f_{3}$ is a finite step function, $\left\|f_{3}\right\|_{\infty} \leq 1$ and the support of $f_{3}$ is contained in a set of measure 1. This however follows from Lemma 2.1.

Thus Theorem 1.1 follows from Lemma 2.1. In the proof of Lemma 2.1 we shall make some finiteness assumptions which we now justify. Fix $f_{1}, f_{2}, f_{3} \in C^{\Delta}\left(\mathbb{R}^{+}\right)$. Then we have

$$
\Lambda_{E}\left(f_{1}, f_{2}, f_{3}\right)=\lim _{K \rightarrow \infty} \int \sum_{k=-K}^{K} \sum_{|\Omega|=2^{1-k}}\left(E_{k-L} \Omega_{u} f_{1}\right)(x) \prod_{j=2}^{3}\left(\overline{\Omega_{l}} f_{j}\right)(x) d x .
$$

Namely, for small values of $k, \Omega_{u} f_{1}$ vanishes if $\Omega=2^{1-k}$, whereas for large values of $k$ the contribution to $\Lambda_{E}\left(f_{1}, f_{2}, f_{3}\right)$ decays geometrically as soon as $\left[0,2^{k-L}\right)$ contains the support of $f_{1}, f_{2}$, and $f_{3}$. Thus it suffices to consider 
sums over finitely many values of $k$ only. For fixed $k$, only finitely many intervals $\Omega$ of length $2^{1-k}$ give a contribution to $\Lambda_{E}\left(f_{1}, f_{2}, f_{3}\right)$. Finally, for fixed $\Omega$, only finitely many tiles $p$ with $\Omega_{p}=\Omega$ give a contribution to $\Omega f_{j}$ and $\bar{\Omega} f_{j}$ because $f_{j}$ is compactly supported for $j=1,2,3$.

In what follows we will assume that all sums involved in (the slightly modified) $\Lambda_{E}$ are finite, but for simplicity of notation we will not adapt our notation to that. We will refer to this reduction as "finite combinatorics assumption" whenever we use it.

\section{The selection of the trees}

A tree consists of the data $T=\left(\xi_{T},\left(F_{k, T}\right)_{k \in \mathbb{Z}}\right)$, where $\xi_{T}>0$ is a frequency and, for each $k, F_{k, T}$ is a set which is the union of dyadic intervals of length $2^{k}$, such that for some $k_{T} \in \mathbb{Z}$ we have $F_{k, T}=\varnothing$ for $k>k_{T}, F_{k_{T}, T}$ is empty or a dyadic interval, and $F_{k, T} \subset F_{k^{\prime}, T}$ whenever $k<k^{\prime} \leq k_{T}$. Given a tree, the number $k_{T}$ is clearly unique unless all $F_{k, T}$ are empty, in which case the choice of $k_{T}$ will be of no importance.

Given a tree $T$ and a $k \in \mathbb{Z}$, we write $\Omega_{k, T}$ for the dyadic interval of length $2^{1-k}$ which contains $\xi_{T}$. We write $\Omega_{k, T, u}$ for the upper half and $\Omega_{k, T, l}$ for the lower half of $\Omega_{k, T}$. Moreover, we define

$$
\Omega_{k, T, y}= \begin{cases}\Omega_{k, T, u} & \text { if } \xi_{T} \in \Omega_{k, T, u} \\ \Omega_{k, T, l} & \text { otherwise. }\end{cases}
$$

(where $y$ stands for "yes, it does contain $\xi_{T}$ "), and we define

$$
\epsilon_{k, T, u}= \begin{cases}1 & \text { if } \xi_{T} \in \Omega_{k, T, u} \\ 0 & \text { otherwise }\end{cases}
$$

and $\epsilon_{k, T, l}=1-\epsilon_{k, T, u}$.

Given data as in Lemma 2.1, we select trees by a recursive algorithm that we now describe. At each step of this algorithm we select a tree and update certain sets $G_{\Omega}^{\nu}$. To begin the algorithm, set $G_{\Omega}^{1}=\mathbb{R}_{0}^{+}$for all dyadic intervals $\Omega$.

Let $\nu \geq 1$ and assume we have already selected the first $\nu-1$ trees, and we have for each dyadic interval $\Omega$ a set $G_{\Omega}^{\nu}$ such that $G_{\Omega}^{\nu}$ is a union of dyadic intervals of length $2|\Omega|^{-1}$ and $G_{\Omega}^{\nu} \subset G_{\Omega^{\prime}}^{\nu}$ if $\Omega^{\prime} \subset \Omega$.

For each pair $I, \xi$ with $I$ a dyadic interval and $\xi$ a positive number define the associated tree to consist of the data $\xi_{T}=\xi, F_{k, T}=I \cap G_{\Omega_{k, T}}^{\nu}$ if $2^{k} \leq|I|$, 
and $F_{k, T}=\varnothing$ if $2^{k}>|I|$, where (matching the previous definition) $\Omega_{k, T}$ denotes the interval of length $2^{1-k}$ which contains $\xi_{T}$.

Let $\mu_{\nu}$ be the largest integer such that there exists a pair $I, \xi$ such that the associated tree $T$ satisfies one of the following eleven conditions (for some $j \in\{2,3\}$ whenever applicable):

$$
\begin{aligned}
& \left\|\epsilon_{k_{T}, T, u} F_{k_{T}, T} E_{k_{T}} \Omega_{k_{T}, T, u} f_{1}\right\|_{2}>2^{\mu_{\nu}}|I|^{\frac{1}{2}}, \\
& \sum_{k}\left\|\epsilon_{k, T, u} F_{k, T} E_{k-L} \overline{\Omega_{k, T, l}} f_{j}\right\|_{2}^{2} \geq 2^{2 \mu_{\nu}}|I|, \\
& \sum_{k}\left\|\epsilon_{k, T, l} F_{k, T} E_{k} \Omega_{k, T, u} f_{1}\right\|_{2}^{2} \geq 2^{2 \mu_{\nu}}|I|, \\
& \sum_{k}\left\|\epsilon_{k+L, T, u} F_{k, T} E_{k} \overline{\Omega_{k+L, T, l}} f_{j}\right\|_{2}^{2} \geq 2^{2 \mu_{\nu}}|I|, \\
& \sum_{k}\left\|\epsilon_{k+L, T, l} F_{k, T} E_{k} \overline{\Omega_{k+L, T, u}} f_{j}\right\|_{2}^{2} \geq 2^{2 \mu_{\nu}}|I|, \\
& \left\|F_{k_{T}, T} E_{k_{T}} \overline{\Omega_{k_{T}+L, T, y}} f_{j}\right\|_{2}>2^{\mu_{\nu}}|I|^{\frac{1}{2}}, \\
& \left\|F_{k_{T}, T} E_{k_{T}-L} \overline{\Omega_{k_{T}, T, y}} f_{j}\right\|_{2} \geq 2^{\mu_{\nu}}|I|^{\frac{1}{2}}, \\
& \left\|F_{k_{T}, T} E_{k_{T}-L+1} \overline{\Omega_{k_{T}+1, T, y}} f_{j}\right\|_{2} \geq 2^{\mu_{\nu}}|I|^{\frac{1}{2}}, \\
& \sum_{k}\left\|F_{k, T}\left(E_{k-L}-E_{k-L+1}\right) \overline{\Omega_{k, T, y}} f_{j}\right\|_{2}^{2} \geq 2^{2 \mu_{\nu}}|I|, \\
& \sum_{k}\left\|\epsilon_{k+1, T, u} F_{k, T} E_{k-L+1} \overline{\Omega_{k+1, T, l}} f_{j}\right\|_{2}^{2} \geq 2^{2 \mu_{\nu}}|I|, \\
& \sum_{k}\left\|\epsilon_{k+1, T, l} F_{k, T} E_{k-L+1} \overline{\Omega_{k+1, T, u}} f_{j}\right\|_{2}^{2} \geq 2^{2 \mu_{\nu}}|I| .
\end{aligned}
$$

(All these conditions are similar, in fact with some additional notation one could reduce the number of conditions here. However, we hope that being explicit here helps with a line-to-line reading of the most involved next section of this paper).

Pick a $\sigma_{\nu} \in\{(3.1), \ldots,(3.11)\}$ such that there is a pair $I, \xi$ such that the associated tree satisfies condition $\sigma_{\nu}$ with the given $\mu_{\nu}$.

If $\sigma_{\nu} \in\{(3.1),(3.6),(3.7),(3.8),(3.9)\}$, pick such a pair $I, \xi$ so that $|I|$ is maximal and call this pair $I_{\nu}, \xi_{\nu}$. If $\sigma \in\{(3.2),(3.4),(3.10)\}$, pick such a pair so that $\xi$ is minimal and call this pair $I_{\nu}, \xi_{\nu}$. If $\sigma \in\{(3.3),(3.5),(3.11)\}$, 
pick such a pair so that $\xi$ is maximal and call this pair $I_{\nu}, \xi_{\nu}$. (By the "finite combinatorics assumption" these maxima and minima can be assumed to be attained.) Let $T_{\nu}$ be the associated tree to the pair $I_{\nu}, \xi_{\nu}$. Define

$$
G_{\Omega}^{\nu+1}:=\left\{\begin{array}{cl}
G_{\Omega}^{\nu} \backslash F_{k, T_{\nu}} & \text { if } \Omega=\Omega_{k, T_{\nu}} \text { for some } k \\
G_{\Omega}^{\nu} & \text { otherwise. }
\end{array}\right.
$$

This completes the $\nu$-th iteration step of the selection algorithm for the trees.

The iteration stops at step $\nu$, when for all pairs $I, \xi$ the left hand side of all conditions (3.1) - (3.11) vanishes. By the "finite combinatorics assumption" this happens for some finite $\nu^{\prime}$. In particular the left hand side of (3.1) vanishes for all pairs $I, \xi$, therefore

$$
G_{\Omega}^{\nu^{\prime}} E_{k} \Omega_{u} f_{1}=0
$$

and therefore

$$
G_{\Omega}^{\nu^{\prime}} E_{k-L} \Omega_{u} f_{1}=0
$$

for all $k$ and $\Omega$ with $|\Omega|=2^{k-1}$.

Therefore we can write

$$
\begin{aligned}
\left|\Lambda_{E}\left(f_{1}, f_{2}, f_{3}\right)\right| & =\left|\int \sum_{k} \sum_{|\Omega|=2^{1-k}} \sum_{\nu=1}^{\nu^{\prime}-1}\left(G_{\Omega}^{\nu}-G_{\Omega}^{\nu+1}\right)\left(E_{k-L} \Omega_{u} f_{1}\right) \prod_{j=2}^{3}\left(\overline{\Omega_{l}} f_{j}\right) d x\right| \\
& =\left|\sum_{T} \int \sum_{\text {selected }} F_{k, T}\left(E_{k-L} \Omega_{k, T, u} f_{1}\right) \prod_{j=2}^{3}\left(\overline{\Omega_{k, T, l}} f_{j}\right) d x\right|
\end{aligned}
$$

By Lemma 4.1 this is bounded by

$$
C_{\delta} \sum_{\nu=1}^{\nu^{\prime}-1} 2^{(3-\delta) \mu_{\nu}}\left|I_{\nu}\right|
$$

for any $\delta>0$. By Lemma 5.1 this is bounded by

$$
C_{\delta} \sum_{\mu \leq 0} 2^{(3-\delta) \mu} 2^{-2 \mu}
$$

This is a bounded geometric series for $\delta<1$. Thus Lemma 2.1 is reduced to Lemmata 4.1 and 5.1. 


\section{The tree estimate}

In this section we prove an estimate on a single selected tree. Let $T_{\nu}$ be one of the selected trees. Since we fix $\nu$ throughout this section, we shall write $T$ for $T_{\nu}, \mu$ for $\mu_{\nu}, I$ for $I_{\nu}$ and $\xi$ for $\xi_{\nu}$. Moreover, we will drop $T$ wherever it appears as an index, e.g. we write $F_{k}$ for $F_{k, T}$ and $\Omega_{k, l}$ for $\Omega_{k, T, l}$. From Lemma 5.1 of the following section we have $\mu \leq 0$.

Lemma 4.1 We have for every $\delta>0$

$$
\left|\sum_{k} \int\left(F_{k} E_{k-L} \Omega_{k, u} f_{1}\right) \prod_{j}\left(\overline{\Omega_{k, l}} f_{j}\right) d x\right| \leq C_{\delta}|I| 2^{(3-\delta) \mu} .
$$

Here the index $j$ runs as always from 2 to 3 . Since $\mu \leq 0$, the estimate becomes sharper when $\delta$ becomes smaller. In this section we will use identities such as $\epsilon_{k, u}=\left(\epsilon_{k, u}\right)^{2}$ and $E_{k-L}=E_{k} E_{k-L}$ without further comment.

We prove the lemma. We insert $1=\epsilon_{k, u}+\epsilon_{k, l}$ into the sum over $k$ and estimate the terms corresponding to $\epsilon_{k, u}$ and $\epsilon_{k, l}$ separately. First we have

$$
\begin{aligned}
& \left|\sum_{k} \int\left(\epsilon_{k, u} F_{k} E_{k-L} \Omega_{k, u} f_{1}\right) \prod_{j}\left(\overline{\Omega_{k, l}} f_{j}\right) d x\right| \leq \\
& \left.\leq\left(\sup _{k}\left\|\epsilon_{k, u} F_{k} E_{k} \Omega_{k, u} f_{1}\right\|_{\infty}\right) \prod_{j}\left(\sum_{k} \| \epsilon_{k, u} F_{k} E_{k-L} \overline{\Omega_{k, l}} f_{j}\right) \|_{2}^{2}\right)^{\frac{1}{2}} .
\end{aligned}
$$

We consider the first factor on the right hand side. Assume we had

$$
\sup _{k}\left\|\epsilon_{k, u} F_{k} E_{k} \Omega_{k, u} f_{1}\right\|_{\infty}>2^{\mu+1} .
$$

Then there was a $k^{\prime} \in \mathbb{Z}$ and a dyadic interval $I^{\prime}$ of length $2^{k^{\prime}}$ contained in $F_{k^{\prime}}$ such that

$$
\left\|\epsilon_{k^{\prime}, u} I^{\prime} E_{k^{\prime}} \Omega_{k^{\prime}, u} f_{1}\right\|_{\infty}>2^{\mu+1} .
$$

Since the function $I^{\prime} E_{k^{\prime}} \Omega_{k^{\prime}, u} f_{1}$ has constant modulus on dyadic intervals of length $2^{k^{\prime}}$, we conclude

$$
\left\|\epsilon_{k^{\prime}, u} I^{\prime} E_{k^{\prime}} \Omega_{k^{\prime}, u} f_{1}\right\|_{2}>2^{\mu+1}\left|I^{\prime}\right|^{\frac{1}{2}} .
$$

This however is impossible, because then at the time $T$ was selected the tree associated to the data $I^{\prime}, \xi$ would have satisfied condition (3.1) with $\mu$ replaced by $\mu+1$, and therefore $T$ could not have been selected. This type of reasoning will occur several times in this section. We will say that a bound 
follows from the tree selection algorithm and we will refer to the selection rule which is used.

Hence (4.1) is bounded by

$$
\left.\leq C 2^{\mu} \prod_{j}\left(\sum_{k} \| \epsilon_{k, u} F_{k} E_{k-L} \overline{\Omega_{k, l}} f_{j}\right) \|_{2}^{2}\right)^{\frac{1}{2}}
$$

By the tree selection algorithm (3.2) it follows that this is bounded by

$$
\leq C 2^{3 \mu}|I|
$$

Since $\mu \leq 0$, this implies the appropriate bound for the term (4.1).

It remains to prove an estimate on

$$
\left|\int \sum_{k}\left(\epsilon_{k, l} F_{k} E_{k-L} \Omega_{k, u} f_{1}\right) \prod_{j}\left(\overline{\Omega_{k, l}} f_{j}\right) d x\right| .
$$

First we observe that (4.2) is equal to

$$
\left|\int \sum_{k}\left(\epsilon_{k, l} F_{k} E_{k} \Omega_{k, u} f_{1}\right)\left(F_{k} E_{k-L} \prod_{j} \overline{\Omega_{k, y}} f_{j}\right) d x\right|
$$

because either $\Omega_{k, l}$ and $\Omega_{k, y}$ coincide or $\epsilon_{k, l}=0$.

We resolve the difference in scales between $\Omega_{k, u}$ and $\overline{\Omega_{k, y}}$ by a telescoping series. Thus we write for the integrand of (4.3)

$$
\begin{gathered}
\sum_{k}\left(\epsilon_{k, l} F_{k} E_{k} \Omega_{k, u} f_{1}\right)\left(F_{k+L} E_{k} \prod_{j} \overline{\Omega_{k+L, y}} f_{j}\right)+ \\
+\sum_{k}\left(\epsilon_{k, l} F_{k} E_{k} \Omega_{k, u} f_{1}\right) \times
\end{gathered}
$$

$$
\times \sum_{\kappa=0}^{L-1}\left[F_{k+\kappa} E_{k-L+\kappa} \prod_{j} \overline{\Omega_{k+\kappa, y}} f_{j}-F_{k+\kappa+1} E_{k-L+\kappa+1} \prod_{j} \overline{\Omega_{k+\kappa+1, y}} f_{j}\right] .
$$

The initial term (4.4) of the telescoping series turns out to be negligible. Namely, the product $\prod_{j} \overline{\Omega_{k+L, y}} f_{j}$ is constant on dyadic intervals of length $2^{k}$. So are the characteristic functions of the sets $E_{k}, F_{k}$, and $F_{k+L}$. But $\Omega_{k, u} f_{1}$ has mean zero on each interval of length $2^{k}$. Hence the integral of (4.4) vanishes, and we can disregard this term in our estimate. 
The telescoping series (4.5) itself simplifies by a change in the order of summation. Thus we write (4.5) as

$$
\begin{gathered}
\sum_{k}\left[\sum_{\kappa=0}^{L-1}\left(\epsilon_{k-\kappa, l} F_{k-\kappa} E_{k-\kappa} \Omega_{k-\kappa, u} f_{1}\right)\right] \times \\
\times\left[F_{k} E_{k-L} \prod_{j} \overline{\Omega_{k, y}} f_{j}-F_{k+1} E_{k-L+1} \prod_{j} \overline{\Omega_{k+1, y}} f_{j}\right] .
\end{gathered}
$$

The difference in (4.7) we resolve further by a short telescoping series as

$$
\begin{aligned}
& {\left[F_{k}\left(E_{k-L}-E_{k-L+1}\right) \prod_{j} \overline{\Omega_{k, y}} f_{j}\right] } \\
+ & {\left[F_{k} E_{k-L+1} \prod_{j}\left(\overline{\Omega_{k, y}} f_{j}-\overline{\Omega_{k+1, y}} f_{j}\right)\right] } \\
+ & {\left[F_{k} E_{k-L+1}\left(\overline{\Omega_{k, y}} f_{2}-\overline{\Omega_{k+1, y}} f_{2}\right) \overline{\Omega_{k+1, y}} f_{3}\right] } \\
+ & {\left[F_{k} E_{k-L+1} \overline{\Omega_{k+1, y}} f_{2}\left(\overline{\Omega_{k, y}} f_{3}-\overline{\Omega_{k+1, y}} f_{3}\right)\right] } \\
+ & {\left[\left(F_{k}-F_{k+1}\right) E_{k-L+1} \prod \overline{\Omega_{k+1, y}} f_{j}\right] . }
\end{aligned}
$$

Here the middle three terms (4.9), (4.10), and (4.11) are not exactly telescoping, but follow from the algebraic identity

$$
a b-A B=(a-A)(b-B)+(a-A) B+A(b-B) .
$$

The terms (4.10) and (4.11) have a mean zero property similar to the one in the discussion of (4.4). We shall only discuss the term (4.10) in detail, because of the symmetry between the terms. We observe that (4.10) has mean zero on dyadic intervals of length $2^{k-L+1}$. Namely, the characteristic functions of $F_{k}$ and $E_{k-L+1}$ are constant on intervals of length $2^{k-L+1}$, whereas

$$
\left(\overline{\Omega_{k, y}} f_{2}-\overline{\Omega_{k+1, y}} f_{2}\right) \overline{\Omega_{k+1, y}} f_{3}=\overline{\left(\Omega_{k, y} \backslash \Omega_{k+1, y}\right)} f_{2} \overline{\Omega_{k+1, y}} f_{3}
$$

is on each interval of length $2^{k-L+1}$ the product of two different (rescaled) Walsh functions and therefore has mean 0. The terms in (4.6) are constant 
on dyadic intervals of length $2^{k-L+1}$ unless $\kappa=L-1$. Thus, after changing the summation index again, it suffices to estimate

$$
\begin{aligned}
& \left|\sum_{k} \int\left(\epsilon_{k, l} F_{k} E_{k} \Omega_{k, u} f_{1}\right)\left(F_{k+L-1} E_{k}\left(\overline{\Omega_{k+L-1, y}} f_{2}-\overline{\Omega_{k+L, y}} f_{2}\right) \overline{\Omega_{k+L, y}} f_{3}\right) d x\right| \\
\leq & \sum_{k} \int\left|\left(\epsilon_{k, l} F_{k} E_{k} \Omega_{k, u} f_{1}\right)\left(F_{k} E_{k}\left(\overline{\Omega_{k+L-1, y}} f_{2}-\overline{\Omega_{k+L, y}} f_{2}\right)\right)\left(F_{k} E_{k} \overline{\Omega_{k+L, y}} f_{3}\right)\right| d x
\end{aligned}
$$

$$
\begin{aligned}
& \leq\left(\sum_{k}\left\|\epsilon_{k, l} F_{k} E_{k} \Omega_{k, u} f_{1}\right\|_{2}^{2}\right)^{\frac{1}{2}} \times \\
& \times\left(\sum_{k}\left\|F_{k} E_{k}\left(\overline{\Omega_{k+L-1, y}} f_{2}-\overline{\Omega_{k+L, y}} f_{2}\right)\right\|_{2}^{2}\right)^{\frac{1}{2}}\left(\sup _{k}\left\|F_{k} E_{k} \overline{\Omega_{k+L, y}} f_{3}\right\|_{\infty}\right) .
\end{aligned}
$$

We observe that

$$
\begin{aligned}
\sum_{k} & \left\|F_{k} E_{k}\left(\overline{\Omega_{k+L-1, y}} f_{2}-\overline{\Omega_{k+L, y}} f_{2}\right)\right\|_{2}^{2}= \\
& =\sum_{k}\left\|\epsilon_{k+L, u} F_{k} E_{k}\left(\overline{\Omega_{k+L, l}} f_{2}\right)\right\|_{2}^{2}+\sum_{k}\left\|\epsilon_{k+L, l} F_{k} E_{k}\left(\overline{\Omega_{k+L, u}} f_{2}\right)\right\|_{2}^{2} .
\end{aligned}
$$

Thus it follows from the selection algorithm (3.3), (3.4), (3.5), (3.6), that (4.13) is bounded by $2^{3 \mu}|I|$.

It remains to discuss (4.8), (4.9), and (4.12). We shall estimate the integrals involving these terms by

$$
\left\|\sup _{k}\left|\sum_{\kappa=0}^{L-1}\left(\epsilon_{k-\kappa, l} F_{k-\kappa} E_{k-\kappa} \Omega_{k-\kappa, u} f_{1}\right)\right|\right\| \prod_{r^{\prime}}\left\|\left(\sum_{k}|[\cdots]|^{2}\right)^{\frac{1}{2}}\right\|_{2 r}
$$

where $[\cdots]$ stands for the expressions (4.8), (4.9), and (4.12) with the product sign $\prod_{j}$ removed. The exponent $r>1$ will be chosen close to 1 and $r^{\prime}$ denotes the conjugate exponent of $r$. In the following calculations constants are allowed to depend on $r$.

We consider the first factor in (4.14). Since $F_{k}$ and $E_{k}$ are unions of dyadic intervals of length $2^{k}$ and $\Omega_{k, u}$ maps into the span of Haar functions 
of scale $2^{k}$, we observe easily with the Hardy Littlewood maximal function

$$
\begin{gathered}
\left\|\sup _{k^{\prime}}\left|\sum_{k>k^{\prime}}\left(\epsilon_{k, l} F_{k} E_{k} \Omega_{k, u} f_{1}\right)\right|\right\|_{r^{\prime}} \leq\left\|M\left(\sum_{k}\left(\epsilon_{k, l} F_{k} E_{k} \Omega_{k, u} f_{1}\right)\right)\right\|_{r^{\prime}} \\
\leq C\left\|\sum_{k}\left(\epsilon_{k, l} F_{k} E_{k} \Omega_{k, u} f_{1}\right)\right\|_{r^{\prime}} \leq C|I|^{\frac{1}{r^{\prime}}}\left\|\sum_{k}\left(\epsilon_{k, l} F_{k} E_{k} \Omega_{k, u} f_{1}\right)\right\|_{\mathrm{BMO}} .
\end{gathered}
$$

In the last inequality we have used that each $F_{k}$ is contained in $I$. In order to estimate the BMO norm let $I^{\prime}$ be any dyadic interval. Then we have on $I^{\prime}$

$$
\sum_{k}\left(\epsilon_{k, l} F_{k} E_{k} \Omega_{k, u} f_{1}\right)=c+\sum_{2^{k} \leq\left|I^{\prime}\right|}\left(\epsilon_{k, l} F_{k} E_{k} \Omega_{k, u} f_{1}\right)
$$

for some number $c$ depending on $I^{\prime}$. Observe that the operators $F_{k} E_{k} \Omega_{k, u}$ have pairwise orthogonal images as $k$ varies. Hence we have

$$
\left\|\sum_{k: 2^{k} \leq\left|I^{\prime}\right|}\left(\epsilon_{k, l} F_{k} E_{k} \Omega_{k, u} f_{1}\right)\right\|_{L^{2}\left(I^{\prime}\right)}^{2}=\sum_{k: 2^{k} \leq\left|I^{\prime}\right|}\left\|\epsilon_{k, l} F_{k} E_{k} \Omega_{k, u} f_{1}\right\|_{L^{2}\left(I^{\prime}\right)}^{2} .
$$

Now we construct a new tree $T^{\prime}$ with $\xi_{T^{\prime}}=\xi$, and $F_{k, T^{\prime}}=I^{\prime} \cap F_{k}$ if $2^{k} \leq\left|I^{\prime}\right|$ and $F_{k, T^{\prime}}=\varnothing$ otherwise. It is easy to see that at the time $T$ was selected, $T^{\prime}$ was the associated tree to the pair $I^{\prime}, \xi^{\prime}$ (here we assume $I^{\prime} \subset I$, the case $I^{\prime} \not \subset I$ is easily done with an $L^{2}$ estimate for $T$ ). Hence we have

$$
\sum_{k: 2^{k} \leq\left|I^{\prime}\right|}\left\|\epsilon_{k, l} F_{k} E_{k} \Omega_{k, u} f_{1}\right\|_{L^{2}\left(I^{\prime}\right)}^{2}=\sum_{k}\left\|\epsilon_{k, T^{\prime}, l} F_{k, T^{\prime}} E_{k} \Omega_{k, T^{\prime}, u} f_{1}\right\|_{2}^{2} .
$$

By the tree selection algorithm (3.3) this is bounded by $C 2^{2 \mu}\left|I^{\prime}\right|$. Thus the above BMO norm is bounded by $C 2^{\mu}$ and we have

$$
\left\|\sup _{k}\left|\sum_{\kappa=0}^{L-1}\left(\epsilon_{k-\kappa, l} F_{k-\kappa} E_{k-\kappa} \Omega_{k-\kappa, u} f_{1}\right)\right|\right\|_{r^{\prime}} \leq C 2^{\mu}|I|^{\frac{1}{r^{\prime}}}
$$

The other factors in (4.14) will be estimated via

$$
\begin{aligned}
& \left\|\left(\sum_{k}|[\cdots]|^{2}\right)^{1 / 2}\right\|_{2 r} \leq\left\|\left(\sum_{k}|[\cdots]|^{2}\right)^{1 / 2}\right\|_{2}^{1 / s}\left\|\left(\sum_{k}|[\cdots]|^{2}\right)^{1 / 2}\right\|_{\frac{2 r s}{s^{\prime}(s-r)}}^{1 / s^{\prime}} \\
& 4.17) \leq C\left\|\left(\sum_{k}|[\cdots]|^{2}\right)^{1 / 2}\right\|_{2}^{1 / s}\left\|\left(\sum_{k}|[\cdots]|^{2}\right)^{1 / 2}\right\|_{\mathrm{BMO}}^{1 / s^{\prime}}|I|^{\frac{1}{2 r}-\frac{1}{2 s}}
\end{aligned}
$$


Here $1<r<s$ and $s$ is chosen close to $r$. The $L^{2}$ norm will be estimated by a good estimate involving $\mu$, whereas for the BMO norm we will only use a crude estimate by a constant. The balance of the two norms is in favor of the good estimate at $L^{2}$, which will make our final argument work.

We consider the case of (4.17) where [ ...] stands for the expression (4.8) with $\prod_{j}$ removed. Observe that we have for the $L^{2}$ norm by the tree selection algorithm (3.9)

$$
\left\|\left(\sum_{k}\left|F_{k}\left(E_{k-L}-E_{k-L+1}\right) \overline{\Omega_{k, y}} f_{j}\right|^{2}\right)^{1 / 2}\right\|_{2} \leq C 2^{\mu}|I|^{\frac{1}{2}}
$$

The BMO norm we estimate by the $L^{\infty}$ norm. Since the sets $E_{k+1} \backslash E_{k}$ are pairwise disjoint, there exists a $k^{\prime}$ and a dyadic interval $I^{\prime}$ of length $2^{k^{\prime}-L+1}$ contained in $E_{k^{\prime}-L+1}$ such that

$$
\left\|\left(\sum_{k}\left|F_{k}\left(E_{k-L}-E_{k-L+1}\right) \overline{\Omega_{k, y}} f_{j}\right|^{2}\right)^{1 / 2}\right\|_{\infty} \leq\left\|I^{\prime} \overline{\Omega_{k^{\prime}, y}} f_{j}\right\|_{\infty} .
$$

Since $\overline{\Omega_{k^{\prime}, y}}$ commutes with $I^{\prime}$ and the modulus of $I^{\prime} \overline{\Omega_{k^{\prime}, y}} f_{j}$ is constant on both dyadic half intervals of $I^{\prime}$, we have

$$
\left\|I^{\prime} \overline{\Omega_{k^{\prime}, y}} f_{j}\right\|_{\infty} \leq 2\left|I^{\prime}\right|^{-\frac{1}{2}}\left\|I^{\prime} \overline{\Omega_{k^{\prime}, y}} f_{j}\right\|_{2} \leq 2 \inf _{x \in I^{\prime}} M_{2} f_{j}(x) \leq 2,
$$

the last inequality by definition of $E_{k^{\prime}-L+1}$. We remark that this argument is the reason for defining $\Lambda_{E}$ with the projection operator $E_{k-L}$ instead of $E_{k}$. Thus we have

$$
\left\|\left(\sum_{k}\left|F_{k}\left(E_{k-L}-E_{k-L+1}\right) \overline{\Omega_{k, y}} f_{j}\right|^{2}\right)^{1 / 2}\right\|_{2 r} \leq C 2^{\frac{\mu}{s}}|I|^{\frac{1}{2 r}}
$$

which together with (4.16) gives the desired estimate for (4.14) in this case provided $s$ is chosen close to 1 .

Next, we consider the case of (4.17) where [...] stands for expression (4.9) with $\prod_{j}$ removed. For the $L^{2}$ norm we observe

$$
\begin{gathered}
\left\|\left(\sum_{k}\left|F_{k} E_{k-L+1}\left(\overline{\Omega_{k, y}} f_{j}-\overline{\Omega_{k+1, y}} f_{j}\right)\right|^{2}\right)^{\frac{1}{2}}\right\|_{2} \\
\leq\left\|\left(\sum_{k}\left|\epsilon_{k+1, u} F_{k} E_{k-L+1} \overline{\Omega_{k+1, l}} f_{j}\right|^{2}\right)^{\frac{1}{2}}\right\|_{2} \\
\quad+\left\|\left(\sum_{k}\left|\epsilon_{k+1, l} F_{k} E_{k-L+1} \overline{\Omega_{k+1, u}} f_{j}\right|^{2}\right)^{\frac{1}{2}}\right\|_{2} .
\end{gathered}
$$


By the selection algorithm (3.10), (3.11) this is bounded by $C 2^{\mu}|I|^{\frac{1}{2}}$. To obtain a BMO estimate fix any dyadic interval $I^{\prime}$. We use the algebraic fact that for $a, b>0$

$$
|a-b|^{2} \leq\left|a^{2}-b^{2}\right|
$$

and the observation that

$$
\left|F_{k} E_{k-L+1}\left(\overline{\Omega_{k, y}} f_{j}-\overline{\Omega_{k+1, y}} f_{j}\right)\right|
$$

is constant on dyadic intervals of length $2^{k-L}$ to obtain

$$
\begin{aligned}
\inf _{c} & \left\|\left(\sum_{k}\left|F_{k} E_{k-L+1}\left(\overline{\Omega_{k, y}} f_{j}-\overline{\Omega_{k+1, y}} f_{j}\right)\right|^{2}\right)^{\frac{1}{2}}-c\right\|_{L^{2}\left(I^{\prime}\right)} \\
& \leq\left\|\left(\sum_{k: 2^{k-L}<\left|I^{\prime}\right|}\left|F_{k} E_{k-L+1}\left(\overline{\Omega_{k, y}} f_{j}-\overline{\Omega_{k+1, y}} f_{j}\right)\right|^{2}\right)^{\frac{1}{2}}\right\|_{L^{2}\left(I^{\prime}\right)} .
\end{aligned}
$$

If $I^{\prime}$ is disjoint from $E$, then it is also disjoint from $E_{k-L+1}$ whenever $2^{k-L}<$ $\left|I^{\prime}\right|$, and therefore the previously displayed expression vanishes. We may therefore assume that there exists an $x \in I^{\prime} \cap E$. Then we estimate the previously displayed expression by

$$
\left\|\left(\sum_{2^{k-L}<\left|I^{\prime}\right|}\left|I^{\prime}\left(\overline{\Omega_{k, y}} f_{j}-\overline{\Omega_{k+1, y}} f_{j}\right)\right|^{2}\right)^{\frac{1}{2}}\right\|_{2} .
$$

The operators $\overline{\Omega_{k, y}}-\overline{\Omega_{k+1, y}}$ in this expression are pairwise orthogonal and commute with $I^{\prime}$, therefore the last expression is bounded by

$$
\left\|I^{\prime} f_{j}\right\|_{2} \leq M_{2} f_{j}(x)\left|I^{\prime}\right|^{\frac{1}{2}} \leq\left|I^{\prime}\right|^{\frac{1}{2}} .
$$

As before this leads to the appropriate bound for (4.14) in this case.

Finally, we consider the case of (4.17) where $[\cdots]$ stands for the expression (4.12) with $\prod_{j}$ removed. Let $\mathbf{I}_{k}$ be the set of dyadic intervals of length $2^{k}$ which have nonempty intersection with $F_{k} \backslash F_{k-1}$. Observe that each interval $I^{\prime} \in \mathbf{I}_{k}$ is contained in $F_{k}$ and intersects $F_{k} \backslash F_{k-1}$ in a set of measure at least $\frac{1}{2}\left|I^{\prime}\right|$. Since the sets $F_{k} \backslash F_{k-1}$ are pairwise disjoint and contained in $I$, we obtain

$$
\sum_{k} \sum_{I^{\prime} \in \mathbf{I}_{k}}\left|I^{\prime}\right| \leq 2|I|
$$

Now observe that for the $L^{2}$ norm we have

$$
\begin{aligned}
& \left\|\left(\sum_{k}\left|\left(F_{k}-F_{k+1}\right) E_{k-L+1} \overline{\Omega_{k+1, y}} f_{j}\right|^{2}\right)^{1 / 2}\right\|_{2}^{2} \\
& \quad \leq\left\|F_{k_{T}} E_{k_{T}-L+1} \overline{\Omega_{k_{T}+1, y}} f_{j}\right\|_{2}^{2}+\sum_{k} \sum_{I^{\prime} \in \mathbf{I}_{k}}\left\|I^{\prime} E_{k-L} \overline{\Omega_{k, y}} f_{j}\right\|_{2}^{2} .
\end{aligned}
$$


By the tree selection algorithm (3.8), (3.7) this is bounded by

$$
\leq C 2^{2 \mu}|I|+C \sum_{k} \sum_{I^{\prime} \in \mathbf{I}_{k}} 2^{2 \mu}\left|I^{\prime}\right| \leq C 2^{2 \mu}|I|
$$

The BMO norm we estimate again by the $L^{\infty}$ norm. Since the sets $F_{k} \backslash F_{k+1}$ are pairwise disjoint, there exists a $k^{\prime}$ and a dyadic interval $I^{\prime}$ of length $2^{k^{\prime}}$ contained in $E_{k^{\prime}}$ such that

$$
\left\|\left(\sum_{k}\left|\left(F_{k}-F_{k+1}\right) E_{k-L+1} \overline{\Omega_{k+1, y}} f_{j}\right|^{2}\right)^{1 / 2}\right\|_{\infty} \leq 2\left\|I^{\prime} \overline{\Omega_{k^{\prime}+L, y}} f_{j}\right\|_{\infty} .
$$

Since the modulus of $I^{\prime} \overline{\Omega_{k^{\prime}+L, y}} f_{j}$ is constant on $I^{\prime}$, we have

$$
\left\|I^{\prime} \overline{\Omega_{k^{\prime}+L, y}} f_{j}\right\|_{\infty}=\left|I^{\prime}\right|^{-\frac{1}{2}}\left\|I^{\prime} \overline{\Omega_{k^{\prime}+L, y}} f_{j}\right\|_{2} \leq \inf _{x \in I^{\prime}} M_{2} f_{j}(x) \leq 1 .
$$

This gives the desired estimate for (4.14) in this case, and therefore completes the discussion of the term (4.14). This also completes the proof of Lemma 4.1 .

\section{Counting the trees}

In this section we prove that there are not too many trees.

Lemma 5.1 We have $\mu_{\nu} \leq 0$ for each selected tree $T_{\nu}$. Moreover

$$
\sum_{\nu: \mu_{\nu}=\mu}\left|I_{\nu}\right| \leq C 2^{-2 \mu}
$$

for every integer $\mu$.

Fix $\mu$ and fix $\sigma \in\{(3.1),(3.2), \ldots,(3.11)\}$. Let $\mathbf{N}$ be the set of indices $\nu$ such that $\mu_{\nu}=\mu$ and $\sigma_{\nu}=\sigma$. We have to prove that

$$
\sum_{\nu \in \mathbf{N}}\left|I_{\nu}\right| \leq C 2^{-2 \mu}
$$

and $\mathbf{N}=\varnothing$ if $\mu>0$. If condition $\sigma$ involves an index $j$, we can also assume $j$ is fixed by splitting into cases.

It is now that we have to pay for having eleven different conditions in the selection algorithm: we have to consider eleven cases for $\sigma$. However, many cases are similar, and we will only do three selected cases explicitly. The necessary modifications to obtain the other cases are easy and essentially consist of replacing indices in the arguments for the selected cases. 
In this section we write $T$ for $T_{\nu}$ and $T^{\prime}$ for $T_{\nu^{\prime}}$.

First consider the case that $\sigma=(3.1)$. For each $\nu \in \mathbf{N}$ condition (3.1) gives

$$
\left\|F_{k_{T}, T} E_{k_{T}} \Omega_{k_{T}, T, y} f_{1}\right\|_{2} \geq 2^{\mu}\left|I_{\nu}\right|^{\frac{1}{2}} .
$$

Here we have used that $\epsilon_{k_{T}, T, u}$ is nonzero and therefore $\Omega_{k_{T}, T, u}=\Omega_{k_{T}, T, y}$. Moreover, (5.2) implies that $F_{k_{T}, T}$ is not empty, therefore it is necessarily equal to $I_{\nu}$. A comparison of spatial scales shows that $\Omega_{k_{T}, T, y}$ commutes with $E_{k_{T}}$ and $I_{\nu}$. In particular we conclude

$$
\left\|I_{\nu} E_{k_{T}} \Omega_{k_{T}, T, y} f_{1}\right\|_{2} \leq\left\|I_{\nu} f_{1}\right\|_{2} \leq\left|I_{\nu}\right|^{\frac{1}{2}} \inf _{x \in I_{\nu}} M_{2} f_{1}(x) \leq\left|I_{\nu}\right|^{\frac{1}{2}} .
$$

Here the last inequality follows from $I_{\nu} \cap E_{k_{T}} \neq \varnothing$, which follows from (5.2). This gives a contradiction to (5.2) unless $\mu \leq 0$.

Now let $\nu, \nu^{\prime} \in \mathbf{N}$. Assume

$$
\left(I_{\nu} \Omega_{k_{T}, T, y}\right)\left(I_{\nu^{\prime}} \Omega_{k_{T^{\prime}}, T^{\prime}, y}\right)^{*} \neq 0 .
$$

Without loss of generality we can assume $k_{T^{\prime}} \leq k_{T}$. Then (5.3) implies $\Omega_{k_{T}, T, y} \subset \Omega_{k_{T^{\prime}}, T^{\prime}, y}$ and $I_{\nu^{\prime}} \subset I_{\nu}$. In particular we can assume that $\nu \leq \nu^{\prime}$, i.e., the tree $T$ has been selected no later than $T^{\prime}$ because at selection step $\nu^{\prime}$ the interval $I_{\nu^{\prime}}$ is maximized. However, $\Omega_{k_{T}, T, y} \subset \Omega_{k_{T^{\prime}, T^{\prime}, y}}$ implies that $\xi_{\nu}$ is contained in $\Omega:=\Omega_{k_{T^{\prime}}, T^{\prime}}$. Hence we have $\Omega=\Omega_{k_{T^{\prime}}, T}$, and since $I_{\nu^{\prime}} \subset I_{\nu}$ we conclude $I_{\nu^{\prime}} \not \subset G_{\Omega}^{\nu+1}$. This implies $\nu^{\prime} \leq \nu$ and therefore $\nu=\nu^{\prime}$.

We conclude that

$$
\left(I_{\nu} \Omega_{k_{T}, T, y}\right)\left(I_{\nu^{\prime}} \Omega_{k_{T^{\prime}}, T^{\prime}, y}\right)^{*}=0
$$

whenever $\nu \neq \nu^{\prime}$, which gives

$$
2^{2 \mu} \sum_{\nu \in \mathbf{N}}\left|I_{\nu}\right| \leq \sum_{\nu \in \mathbf{N}}\left\|I_{\nu} E_{k_{T}} \Omega_{k_{T}, T, y} f_{1}\right\|_{2}^{2} \leq\left\|f_{1}\right\|_{2}^{2} \leq 1 .
$$

(Here we have also used that each operator $I_{\nu} E_{k_{T}} \Omega_{k_{T}, T, y}$ obviously has operator norm bounded by 1.) This proves estimate (5.1) in the case $\sigma=(3.1)$. The cases $\sigma=(3.6),(3.7),(3.8)$ are done similarly.

Next, we consider the case $\sigma=(3.2)$. For every $\nu \in \mathbf{N}$ we have the inequality

$$
\sum_{k}\left\|\epsilon_{k, T, u} F_{k, T} E_{k-L} \overline{\Omega_{k, T, l}} f_{j}\right\|_{2}^{2} \geq 2^{2 \mu}\left|I_{\nu}\right|
$$


Again we observe that $\overline{\Omega_{k, T, l}}$ commutes with the operators $F_{k, T}$ and $E_{k-L}$.

Now let $k, k^{\prime} \in \mathbb{Z}$ and $\nu, \nu^{\prime} \in \mathbf{N}$ and assume that

$$
\left(\epsilon_{k, T, u} F_{k, T} \overline{\Omega_{k, T, l}}\right)\left(\epsilon_{k^{\prime}, T^{\prime}, u} F_{k^{\prime}, T^{\prime}} \overline{\Omega_{k^{\prime}, T^{\prime}, l}}\right)^{*} \neq 0 .
$$

We claim that this implies $k=k^{\prime}$ and $\nu=\nu^{\prime}$. Observe that since $\epsilon_{k, T, u}$ is nonzero, $\xi_{\nu}$ is contained in $\Omega_{k, T, u}$ and lies above $\Omega_{k, T, l}$. The analogue statement holds for $\xi_{\nu^{\prime}}$.

Now assume without loss of generality that $\nu \leq \nu^{\prime}$. Then $\xi_{\nu} \leq \xi_{\nu^{\prime}}$ since small $\xi_{\nu}$ are selected first. Since $\xi_{\nu}$ is above $\Omega_{k, T, l}$, this implies that $\xi_{\nu^{\prime}}$ is not contained in $\Omega_{k, T, l}$. Hence $\Omega_{k^{\prime}, T^{\prime}}$ is not contained in $\Omega_{k, T, l}$ and therefore $\Omega_{k^{\prime}, T^{\prime}, l}$ is not strictly contained in $\Omega_{k, T, l}$. By (5.5) we conclude that $\Omega_{k, T, l}$ has nonempty intersection with $\Omega_{k^{\prime}, T^{\prime}, l}$ and therefore is contained in $\Omega_{k^{\prime}, T^{\prime}, l}$. In particular $k^{\prime} \leq k$.

Moreover, $\Omega:=\Omega_{k^{\prime}, T^{\prime}}$ contains $\xi_{\nu}$. Therefore $\Omega=\Omega_{k^{\prime}, T}$. But (5.5) implies that $F_{k^{\prime}, T^{\prime}}$ and $F_{k, T}$ are not disjoint, hence $F_{k^{\prime}, T^{\prime}}$ and $I_{\nu}$ are not disjoint, hence $G_{\Omega}^{\nu^{\prime}}$ and $I_{\nu}$ are not disjoint. This implies $\nu^{\prime} \leq \nu$ and therefore $\nu=\nu^{\prime}$.

We have seen that $\nu \leq \nu^{\prime}$ implies $k^{\prime} \leq k$; now that we also have $\nu^{\prime} \leq \nu$ we obtain by the same argument $k \leq k^{\prime}$ and hence $k=k^{\prime}$.

This proves the above claim, and we therefore obtain

$$
2^{2 \mu} \sum_{\nu \in \mathbf{N}}\left|I_{\nu}\right| \leq \sum_{\nu \in \mathbf{N}} \sum_{k}\left\|\epsilon_{k, T, u} F_{k, T} E_{k-L} \overline{\Omega_{k, T, l}} f_{j}\right\|_{2}^{2} \leq\left\|f_{j}\right\|_{2}^{2}=1
$$

Moreover, we have for a single $\nu$, since $F_{k, T}=I_{\nu} F_{k, T}$,

$$
\sum_{k}\left\|\epsilon_{k, T, u} F_{k, T} E_{k-L} \overline{\Omega_{k, T, l}} f_{j}\right\|_{2}^{2} \leq\left\|I_{\nu} f_{j}\right\|_{2}^{2} \leq\left|I_{\nu}\right|^{\frac{1}{2}}
$$

because (5.4) implies that $I_{\nu}$ intersects $E$ nontrivially. Thus $\mathbf{N} \neq 0$ implies $\mu \leq 0$.

This completes the discussion of the case $\sigma=(3.2)$. The cases $\sigma=$ (3.4), (3.10) are done similarly. So are the cases $\sigma=(3.3),(3.5),(3.11)$ using the opposite orientation of the frequency axis.

It remains to discuss the case $\sigma=(3.9)$.

For every $\nu \in \mathbf{N}$ we have the inequality

$$
\sum_{k}\left\|F_{k, T}\left(E_{k-L}-E_{k-L+1}\right) \overline{\Omega_{k, T, y}} f_{j}\right\|_{2}^{2} \geq 2^{2 \mu}\left|I_{\nu}\right|
$$


Again we observe that $\overline{\Omega_{k, T, y}}$ commutes with the operators $E_{k-L}-E_{k-L+1}$ and $F_{k, T}$. Now let $k, k^{\prime} \in \mathbb{Z}$ and $\nu, \nu^{\prime} \in \mathbf{N}$ and assume that

$$
\left(F_{k, T}\left(E_{k-L}-E_{k-L+1}\right) \overline{\Omega_{k, T, y}}\right)\left(F_{k^{\prime}, T^{\prime}}\left(E_{k^{\prime}-L}-E_{k^{\prime}-L+1}\right) \overline{\Omega_{k^{\prime}, T^{\prime}, y}}\right)^{*} \neq 0 .
$$

Thus $\left(E_{k-L}-E_{k-L+1}\right)$ and $\left(E_{k^{\prime}-L}-E_{k^{\prime}-L+1}\right)$ have nonempty intersection, which implies $k=k^{\prime}$ by the nesting property (2.3). Since also $\overline{\Omega_{k, T, y}}$ and $\overline{\Omega_{k^{\prime}, T^{\prime}, y}}$ have nonempty intersection, they are equal, and since $F_{k, T}$ and $F_{k^{\prime}, T^{\prime}}$ have nonempty intersection we conclude $\nu=\nu^{\prime}$.

Therefore we obtain as before

$$
2^{2 \mu} \sum_{\nu \in \mathbf{N}}\left|I_{\nu}\right| \leq \sum_{\nu \in \mathbf{N}} \sum_{k}\left\|F_{k, T}\left(E_{k-L}-E_{k-L+1}\right) \overline{\Omega_{k, T, y}} f_{j}\right\|_{2}^{2} \leq\left\|f_{j}\right\|_{2}^{2}=1 .
$$

Moreover, we have for a single $\nu$

$$
\sum_{k}\left\|F_{k, T}\left(E_{k-L}-E_{k-L+1}\right) \overline{\Omega_{k, T, y}} f_{j}\right\|_{2}^{2} \leq\left\|I_{\nu} f_{j}\right\|_{2}^{2} \leq\left|I_{\nu}\right|^{\frac{1}{2}}
$$

Here we have used that $I_{\nu}$ intersects $E$ nontrivially. Namely, (5.6) implies that $I_{\nu}$ intersects $E_{k-L+1} \backslash E_{k-L}$ nontrivially for some $k \leq k_{T}$. Hence it intersects a dyadic interval $I^{\prime} \subset E_{k-L+1}$ of length $2^{k-L+1}$ nontrivially. But since $\left|I^{\prime}\right|<\left|I_{\nu}\right|$ we have $I^{\prime} \subset I_{\nu}$, and by definition $E \cap I^{\prime} \neq \varnothing$.

As before we conclude that $\mathbf{N} \neq 0$ implies $\mu \leq 0$.

This completes the discussion of the case $\sigma=(3.9)$ and therefore also the proof of Lemma 5.1.

\section{References}

[1] Billard, P., Sur la convergence presque partout des series de Fourier Walsh des fonctions de l'espace $L^{2}(0,1)$. Studia Math. 28 (1967), pp. 363-388.

[2] Carleson, L., On convergence and growth of partial sums of Fourier series. Acta Math. 116 (1966) pp. 135-157.

[3] Grafakos, L., Li, X., Uniform bounds for the bilinear Hilbert transforms I. preprint (2000).

[4] Lacey, M., The bilinear maximal function maps into $L^{p}$ for $2 / 3<p \leq 1$. Ann. of Math. 151 (2000), no. 1, pp. 35-57.

[5] Lacey, M., Thiele, C., $L^{p}$ estimates on the bilinear Hilbert transform for $2<p<\infty$. Ann. of Math. 146 (1997), 693-724.

[6] Lacey, M., Thiele, C., Bounds for the bilinear Hilbert transform on $L^{p}$. Proc. Natl. Acad. Sci. USA 94 (1997), pp. 33-35. 
[7] Lacey, M., Thiele, C., On Calderon's conjecture. Ann. of Math. 149 (1999), pp. 475-496.

[8] Lacey, M., Thiele, C., On the Calderon Conjectures for the Bilinear Hilbert Transform. Proc. Natl. Acad. Sci. USA 95 (1998), pp. 4828-4830.

[9] LI, X. Uniform bounds for the bilinear Hilbert transforms II, preprint (2000)

[10] Thiele, C., Time-frequency analysis in the discrete phase plane. $\mathrm{Ph}$. D. thesis, 1995.

[11] Thiele, C., The quartile operator and pointwise convergence of Walsh series. Trans. Amer. Math. Soc. 352 (2000), no. 12, pp. 5745-5766.

[12] Thiele, C. The Maximal Quartile Operator, Rev. Mat. Iberoamericana 17 (2001), 107-135.

[13] Thiele, C. On the Bilinear Hilbert Transform, Habilitationsschrift, Universität Kiel, 1998.

Recibido: 16 de junio de 1999

Christoph Thiele

Department of Mathematics

University of California Los Angeles

Los Angeles, CA 90095-1555

USA

thiele@math.ucla.edu 